\title{
Mathematical modelling, analysis and simulation of the spread of gangs in interacting youth and adult populations
}

\author{
M. Rivera-Castro ${ }^{a}$, P. Padmanabhan ${ }^{b}$, C. Caiseda ${ }^{a}$, P. Seshaiyer ${ }^{c}$ and C. Boria-Guanilla \\ a Department of Natural Sciences and Mathematics, InterAmerican University of Puerto Rico, Bayamon, \\ Puerto Rico; ${ }^{b}$ Dietrich School of Arts and Sciences, University of Pittsburgh, Pittsburgh, PA, USA; ${ }^{c}$ Department \\ of Mathematical Sciences, George Mason University, Fairfax, VA, USA
}

\begin{abstract}
Youth that live in public housing communities with high social and financial needs are at risk of getting involved in illegal drug trafficking gangs that are controlled by adults. This social disease spreads like an epidemic in these densely populated sectors and metropolitan area. In this work, a model based on SIR disease dynamics is used to study the spread of gangs in vulnerable youth and adult sub-populations. Three types of mixing patterns govern interaction between the groups: proportionate, preferred and like-with-like. This new model is analysed presenting formulations for the reproduction number, sensitivity analysis, and stability analysis for the like-withlike mixing. Insights gained from simulation results on the sensitivity of the model to parameters show the relevance of the activity parameter over the reproduction number when heterogeneous mixing is present.
\end{abstract}

\section{ARTICLE HISTORY}

Received 10 December 2018

Accepted 17 June 2019

\section{KEYWORDS}

Mixing patterns; criminal gang; reproduction number; stability analysis; SIR; modelling and simulation

\section{Introduction}

The spread of crime has been modelled as a disease in past work (McMillon, Simon, \& Morenoff, 2014; Mohammad \& Roslan, 2017; Sooknanan, Bhatt, \& Comissiong, 2013) producing multi-compartmental models of a homogeneous population that are analysed to gain insight on fighting this costly social problem in the U.S. and abroad. J. Sooknanan et al. present a dynamical model of the formation of a gang that introduces a non-susceptible as well as the susceptible group. In this onset stage when non-susceptible, susceptible and gang members interact, prevention and intervention parameters are shown to significantly impact gang membership. Our work considers the dynamics of gang formation in a susceptible heterogeneous population that is divided at onset in two groups: youth and adults. The model is motivated by the critical social problem of youth joining illegal drug gangs operating in the U.S. territory of Puerto Rico (PR) (Torres-Gotay, 2017). These gangs are responsible for the great loss of young people into a life of crime due to recruitment into drug trafficking gangs lead by adults. This situation has been recently exacerbated. A struggling economy, a massive exodus of Puerto Rican families to the mainland, a shrinking police force (Levantesi, 2018) and the aftermath of a deadly hurricane that hit the island 
in September 2017 (Silverstein, 2018) have contributed to the rise of drug-related criminal activity ((Chakraborti, 2018), Fox News, CBS News, Center for investigative journalism).

In 2002 a government-funded study on juvenile delinquency and illegal drug trafficking in PR was published by the Office of Youth Affairs. According to the study, adult gang members will recruit socially vulnerable youth that have financial needs, have dropped out of school and therefore have limitations to earn money legally (Muñoz-González, 2007). They are trained as gunmen, watchmen, salesman, and to transport drugs. This study estimated that police interventions with minors from seven to seventeen years-old were 2257 per 100,000 population, higher than the 1857 per 100,000 adult interventions. According to an ex-gang member interviewed in this work, children as young as 11 years-old are seen in drug-related gangs. Data from the 2016 Police Department Census show that there are 542 active drug hot-spots, and $80 \%$ of these use minors, a form of human trafficking (Rey-Hernandez \& Hernandez-Angueira, 2014). Most of the drug hot-spots are located in public housing projects that offer the needed protection for criminal activity of gangs, and therefore operate in neighbourhoods where many vulnerable children live.

Minors are an asset for criminal gangs because the consequences of criminal behaviour are necessarily more lenient for youth than adults, as dictated by the juvenile justice system. In some exceptional cases, a 14-18 year old could be tried as an adult because of the severity of the crime, history and personality factors (Minor's Court, 2018). A minor, according to Minor's Law (Law 88) in PR is defined as an individual under the age of 18. Minors that face charges are tried in Minor's Court that responds to a philosophy of rehabilitation and protection of the minor as a developing citizen. Most offenses will not go to trial but to a Diversion Programme. This Programme is determined by social worker recommendations and negotiation between the legal defense and the Minor Procurator (i.e. the prosecutor). In the rare cases when a minor is trialed and convicted of a major fault, the sentence will have a maximum period of three years in a Juvenile Detention Center. After this, almost all return to criminal activities and are commended by gang leaders.

The fundamental Susceptible-Infected-Recovered SIR model (Kermack \& McKendrick, 1991a, 1991b) has been enhanced to express the contagion between two groups in diseases transmitted by a vector to humans, and via sexual transmission such as the Zika virus (ZIKV) (Padmanabhan, Seshaiyer, \& Castillo-Chavez, 2017). Similarly in the study of heterogeneous gangs considered in this work the interaction between sub-populations or mixing is of great importance. In the gang model an individual may be 'infected' by becoming recruited into the gang by interaction with an adult or youth. Therefore, parameters that control the level of activity and interactions among heterogeneous groups will significantly affect the gang formation. In the next section, we introduce the mathematical model.

\section{Mathematical models and methods}

In this section, we will describe the formulation of models with two sub-populations, youth and adults, within a community with no previous drug-dealing activity. We define the 'Youth' population as vulnerable individuals who are children or adolescents 17 years old or younger when gang recruitment initiated, and are considered from the onset as potentially loyal recruits. This group functions as a 'circle of trust' and continues to be identified as 'youth' through time. Individuals eighteen and older at gang formation are in the adult 
group, and also are considered trustworthy as potential recruits from onset. Drug-related gangs considered in this work are organized crime groups that may have more than 100 members that are recruiting during their criminal activity in all roles, including cover-up and support gang activities. We will assume that the youth sub-population makes contact at a higher rate than adults in the developed model for the spread of gangs.

The Susceptible-Gang-Removed (SGR) model accounts for gang members that continue to recruit susceptible individuals unless they are incarcerated (removed), or leave criminal activity via interventions returning to the susceptible group. Although gang recruitment within correctional facilities is acknowledged, in this model recruitment occurs only from a particular susceptible community where a new drug hot-spot will begin illegal activities. After completing their detention period individuals may go back to criminal activity (recidivism) or, by rehabilitation, go back to the susceptible group. We consider the youth and adult sub-populations of constant sizes $N_{y}$ and $N_{A}$ respectively, each divided into Susceptible ( $S$ : potential to be recruited to a gang), infectious ( $G$ : gang members) and removed ( $R$ : gang members who are caught, serve a detention sentence, or are permanently removed such as life-in-prison or death). Subscripts serve to identify the respective subpopulation. We will assume that the group $y$ members make $a_{1}$ contacts in unit time and group $A$ members make $a_{2}$ contacts in unit time. We will now define fraction of interactions made between the two sub-populations as follows:

$\beta_{y y} \quad$ youth member with another youth member

$\beta_{A A}$ adult member with another adult member

$\beta_{y A} \quad$ youth member with an adult member

$\beta_{A y} \quad$ adult member with a youth member

Note that,

$$
\beta_{y y}+\beta_{y A}=\beta_{A y}+\beta_{A A}=1
$$

Let us define the mean infectious period that in youth population is $1 / \alpha_{y}$ and in adult population is $1 / \alpha_{A}$. Then, we will assume that gang members from both classes $G_{y}$ and $G_{A}$ may be removed (death or prison) with a removal rate of $\alpha_{y}$ and $\alpha_{G}$ respectively. Let $1 / \rho_{y}$ and $1 / \rho_{A}$ be the average length of the jail sentence divided by the percentage of living removed individuals that are serving a non-life in prison sentence, youth or adult group respectively. The proportion of prisoners moving back to becoming a gang member after release is defined as $\left(1-f_{y}\right)=\bar{f}_{y}$ and $\left(1-f_{A}\right)=\bar{f}_{A}$ for youth and adults respectively. Thus $\bar{f}$ denotes the recidivism rate while $f_{y}$ and $f_{A}$ denote the rehabilitation rate for the respective sub-population. Hence in our model, we will assume that a fraction $\bar{f}_{y}$ of the released population may relapse to the class of youth Gang members $G_{y}$ and $f_{y}$ may return to the susceptible youths $S_{y}$. Similarly, we will assume that a fraction $\overline{f_{A}}$ of released adults may relapse to the class of adult Gang members $G_{A}$ and the remaining $f_{A}$ may return to the susceptible adults $S_{A}$. Finally, we will also assume that it is possible to intervene at the infected stage (when part of a gang) for both youth and adults with the intervention parameter $\phi$ ( $\phi_{y}$ for youth and $\phi_{A}$ for adults) and sway youth and adults back into the respective Susceptible classes. For example, such intervention may involve education and counselling. Figure 1 summarizes the interaction between adults and youth described. 


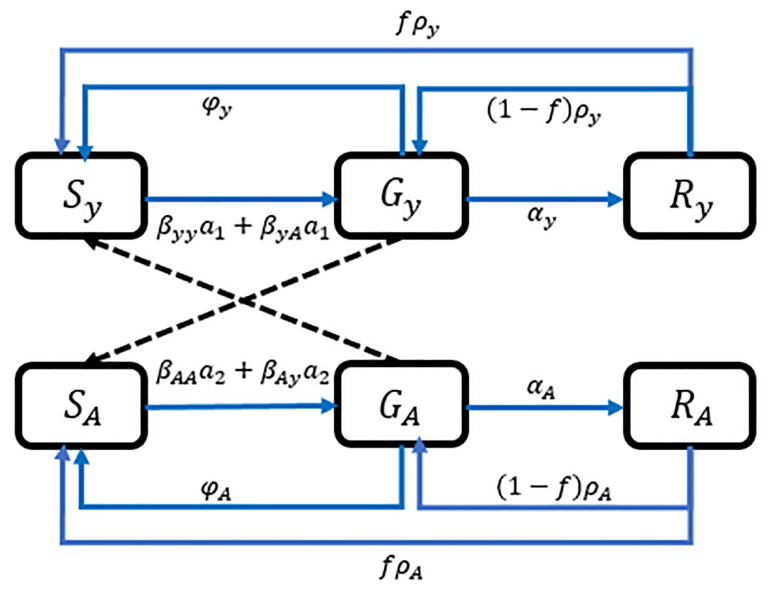

Figure 1. Gang model compartmental diagram.

With these assumptions and notations, our governing system of differential equations describing the spread of gangs for youth and adults may be given as

Youth and Adult Population Equations

$$
\begin{aligned}
\frac{\mathrm{d} S_{y}}{\mathrm{~d} t} & =-\left[\beta_{y y} a_{1}\left(\frac{S_{y} G_{y}}{N_{y}}\right)+\beta_{y A} a_{1}\left(\frac{S_{y} G_{A}}{N_{A}}\right)\right]+f_{y} \rho_{y} R_{y}+\phi_{y} G_{y}, \\
\frac{\mathrm{d} G_{y}}{\mathrm{~d} t} & =\beta_{y y} a_{1}\left(\frac{S_{y} G_{y}}{N_{y}}\right)+\beta_{y A} a_{1}\left(\frac{S_{y} G_{A}}{N_{A}}\right)+\bar{f}_{y} \rho_{y} R_{y}-\phi_{y} G_{y}-\alpha_{y} G_{y}, \\
\frac{\mathrm{d} R_{y}}{\mathrm{~d} t} & =\alpha_{y} G_{y}-\rho_{y} R_{y} \\
\frac{\mathrm{d} S_{A}}{\mathrm{~d} t} & =-\left[\beta_{A A} a_{2}\left(\frac{S_{A} G_{A}}{N_{A}}\right)+\beta_{A y} a_{2}\left(\frac{S_{A} G_{y}}{N_{y}}\right)\right]+f_{A} \rho_{A} R_{A}+\phi_{A} G_{A}, \\
\frac{\mathrm{d} G_{A}}{\mathrm{~d} t} & =\beta_{A A} a_{2}\left(\frac{S_{A} G_{A}}{N_{A}}\right)+\beta_{A y} a_{2}\left(\frac{S_{A} G_{y}}{N_{y}}\right)+\overline{f_{A}} \rho_{A} R_{A}-\phi_{A} G_{A}-\alpha_{A} G_{A}, \\
\frac{\mathrm{d} R_{A}}{\mathrm{~d} t} & =\alpha_{A} G_{A}-\rho_{A} R_{A} .
\end{aligned}
$$

\section{Mathematical analysis}

In the infectious diseases literature, the basic reproduction number $\mathcal{R}_{0}$ denotes the number of secondary infections generated by an infected human when the population being considered is composed of primarily susceptible humans. Here one may analogously consider $\mathcal{R}_{0}$ to represent the number of new gang recruits that occurs when one current youth (or adult) gang member enters the population of a wholly susceptible youth (or adult) population. $\mathcal{R}_{0}$ therefore determines whether there is a continued increase in the number of youth and adult gang members or not. 
In this section, we derive the basic reproduction number $\mathcal{R}_{0}$ for our mathematical model (1)-(6) using the Next Generation Matrix approach (Driessche \& Watmough, 2002) which is described in the theorem next.

Theorem 3.1: The basic reproduction number $\mathcal{R}_{0}$ is given by

$$
\mathcal{R}_{0}=\left(\frac{1}{2}\right)\left[\left(R_{0}^{A A}+R_{0}^{y y}\right)+\sqrt{\left(R_{0}^{y y}-R_{0}^{A A}\right)^{2}+4 R_{0}^{y A} R_{0}^{A y}}\right],
$$

where

$$
\begin{aligned}
R_{0}^{A A} & =\frac{\beta_{A A} a_{2}}{\left(\phi_{A}+\alpha_{A}\right)}, \\
R_{0}^{y y} & =\frac{\beta_{y y} a_{1}}{\left(\phi_{y}+\alpha_{y}\right)}, \\
R_{0}^{y A} & =\frac{\beta_{y A} a_{1}}{\left(\phi_{y}+\alpha_{y}\right)}, \\
R_{0}^{A y} & =\frac{\beta_{A y} a_{2}}{\left(\phi_{A}+\alpha_{A}\right)} .
\end{aligned}
$$

\section{Proof:}

$$
\begin{aligned}
& \mathcal{F}=\left\{\beta_{y y} a_{1} G_{y}+\beta_{y A} a_{1} G_{A} \frac{N_{y}}{N_{A}}, \beta_{A A} a_{2} G_{A}+\beta_{A y} a_{2} G_{y} \frac{N_{A}}{N_{y}}\right\}, \\
& \mathcal{V}=\left\{\left(\phi_{y}+\alpha_{y}\right) G_{y},\left(\phi_{A}+\alpha_{A}\right) G_{A}\right\} .
\end{aligned}
$$

Next, we compute the Jacobian $F$ from $\mathcal{F}$ given by

$$
F=\left(\begin{array}{cc}
\beta_{y y} a_{1} & \beta_{y A} a_{1} \frac{N_{y}}{N_{A}} \\
\beta_{A y} a_{2} \frac{N_{A}}{N_{y}} & \beta_{A A} a_{2}
\end{array}\right)
$$

and the Jacobian $V$ from $\mathcal{V}$ given by

$$
V=\left(\begin{array}{cc}
\phi_{y}+\alpha_{y} & 0 \\
0 & \phi_{A}+\alpha_{A}
\end{array}\right)
$$

Using matrices $F$ and $V$ one can then compute the Next Generation Matrix $F V^{-1}$.

Substituting $t_{y}=\phi_{y}+\alpha_{y}$ and $t_{A}=\phi_{A}+\alpha_{A}$ for simplicity, this matrix can be calculated to be

$$
F V^{-1}=\left(\begin{array}{cc}
\frac{\beta_{y y} a_{1}}{t_{y}} & \frac{\beta_{y A} a_{1} N_{y}}{N_{A} t_{A}} \\
\frac{\beta_{A y} a_{2} N_{A}}{N_{y} t_{y}} & \frac{\beta_{A A} a_{2}}{t_{A}}
\end{array}\right) .
$$

Note that $(i, j)$ entry of the Next Generation Matrix $F V^{-1}$ is the expected number of secondary infections in compartment $i$ produced by individuals initially in compartment $j$ 
assuming that the environment seen by the individual remains homogeneous for the duration of its infection. Also, matrix $F V^{-1}$ is non-negative and therefore has a non-negative eigenvalue. The basic reproduction number can then be computed as $\mathcal{R}_{0}=\rho\left(F V^{-1}\right)$ which is the spectral radius of the matrix. This eigenvalue is associated with a nonnegative eigenvector which represent the distribution of infected individuals that produces the greatest number $\mathcal{R}_{0}$ of secondary infections per generation. In order to calculate the eigenvalues of $F V^{-1}$, we consider the characteristic equation

$$
\operatorname{det}\left(F V^{-1}-\lambda I\right)=0,
$$

where $\lambda$ denotes the eigenvalues of the matrix and $I$ represents the Identity matrix. This can be simplified to yield

$$
\left|\begin{array}{cc}
\frac{\beta_{y y} a_{1}}{t_{y}}-\lambda & \frac{\beta_{y A} a_{1} N_{y}}{N_{A} t_{A}} \\
\frac{\beta_{A y} a_{2} N_{A}}{N_{y} t_{y}} & \frac{\beta_{A A} a_{2}}{t_{A}}-\lambda
\end{array}\right|=0 .
$$

The characteristic polynomial therefore is the following quadratic equation given by

$$
\lambda^{2}-\lambda\left(\frac{\beta_{A A} a_{2}}{t_{A}}+\frac{\beta_{y y} a_{1}}{t_{y}}\right)+\left(\beta_{y y} \beta_{A A}-\beta_{y A} \beta_{A y}\right)\left(\frac{a_{1} a_{2}}{t_{A} t_{y}}\right)=0 .
$$

The basic reproduction number $\mathcal{R}_{0}$ corresponds to the dominant eigenvalue given by the root of the quadratic equation:

$$
\mathcal{R}_{0}=\left(\frac{1}{2}\right)\left[\left(\frac{\beta_{A A} a_{2}}{t_{A}}+\frac{\beta_{y y} a_{1}}{t_{y}}\right)+\sqrt{\left(\frac{\beta_{y y} a_{1}}{t_{y}}-\frac{\beta_{A A} a_{2}}{t_{A}}\right)^{2}+4\left(\frac{\beta_{y A} a_{2}}{t_{A}} \frac{\beta_{A y} a_{1}}{t_{y}}\right)}\right] .
$$

Substituting back in $\phi_{y}+\alpha_{y}$ and $\phi_{A}+\alpha_{A}$ for $t_{y}$ and $t_{A}$ respectively proves the result.

\subsection{Heterogeneous mixing}

In order to obtain a more useful expression for $\mathcal{R}_{0}$, it is necessary to make some assumptions about the nature of the mixing between the groups using structured mixing (Simon $\&$ Jacquez, 1992). The mixing is determined by two quantities $\beta_{y A}$ (infection rate as youth interacts with adults) $\beta_{A y}$ (infection rate as adult interacts with youth) since $\beta_{y y}=1-\beta_{y A}$ and $\beta_{A A}=1-\beta_{A y}$. Interactions between youth and adults in a gang are described as proportionate, preferred and like-with-like mixing (Brauer, 2008; Choe \& Lee, 2015). These describe a progression from the probability of youth and adults interacting randomly, to increasingly homogeneous interactions where individuals prefer contacts within their age group. Proportionate mixing functions define the probability of sub-populations randomly interacting with each other. Preferred mixing identifies a fraction of the interactions that will only occur between the same age group denoted as $\pi_{y}$ for youth and $\pi_{A}$ adults. The remaining activity $\left(1-\pi_{y}\right)$ and $\left(1-\pi_{A}\right)$ will be proportionate. Finally like-with-like mixing refers to interaction exclusively within the same sub-population. In this restricted contact, youth interact exclusively with youth, and consequently adults only contact adults. 
All three mixing types can be obtained from the following formulation by choosing the appropriate value for $\pi_{i} \in[0,1], i \in\{y, A\}$

$$
\begin{aligned}
& \beta_{i i}=\pi_{i}+\left(1-\pi_{i}\right) \beta_{i}, \\
& \beta_{i j}=\left(1-\pi_{i}\right) \beta_{j}, \\
& \beta_{i}=\frac{\left(1-\pi_{i}\right) a_{i} N_{i}}{\left(1-\pi_{y}\right) a_{1} N_{y}+\left(1-\pi_{A}\right) a_{2} N_{A}}, \quad\left(\pi_{y}, \pi_{A}\right) \neq(1,1) .
\end{aligned}
$$

For proportionate mixing of sub-population $i$, let $\pi_{i}=0$. Preferred mixing can be obtained taking $0<\pi_{i}<1$. For like-with-like mixing take only one sub-population $i$ to be $\pi_{i}=1$. This implies like-with-like mixing for population $j$ because for all $\pi_{j} \neq 1, \beta_{i}=0, \beta_{j}=1$ and therefore $\beta_{j, i}=0, \beta_{j, j}=1$. The following corollaries follow from the main Theorem 3.1 for $\mathcal{R}_{0}$.

Corollary 3.2: If adults or youth interact in like-with-like mixing, then

$$
\mathcal{R}_{0}= \begin{cases}R_{0}^{y y}, & R_{0}^{y y} \geq R_{0}^{A A}, \\ R_{0}^{A A}, & R_{0}^{y y}<R_{0}^{A A} .\end{cases}
$$

Proof: For this mixing we have $\pi_{i}=1, \pi_{j} \neq 1$. Note that $R_{0}^{A y}=R_{0}^{y A}=0$ in (7), and therefore by substituting in the $\mathcal{R}_{0}$ formulation from Theorem 1, we get the simple form $\mathcal{R}_{0}=R_{0}^{y y}+R_{0}^{A A}+\left|R_{0}^{y y}-R_{0}^{A A}\right|$.

Corollary 3.2 translates to the notion that when sub-populations interact homogeneously the group with the largest recruiting activity should be the focus of intervention. Treatment of this sub-population that include either decreasing their activity or increasing detention and intervention parameters $\alpha$ and $\phi$ will thwart the gang spread.

Corollary 3.3: If adults and youth both interact in proportionate mixing, then $\mathcal{R}_{0}=R_{0}^{y y}+$ $R_{0}^{A A}$.

Proof: For proportionate mixing we have $\pi_{i}=0$. Therefore, $\beta_{A A}=\beta_{y A}=\beta_{A}$, and $\beta_{A y}=$ $\beta_{y y}=\beta_{y}$. Note that $R_{0}^{A y} R_{0}^{y A}=R_{0}^{y y} R_{0}^{A A}$ in (7). Then the discriminant

$$
\left(R_{0}^{y y}-R_{0}^{A A}\right)^{2}+4 R_{0}^{y A} R_{0}^{A y}
$$

reduces to

$$
\left(R_{0}^{y y}+R_{0}^{A A}\right)^{2} .
$$

The result is obtained by taking the square root and substituting in $\mathcal{R}_{0}$ from Theorem 1 .

From Corollary 3.3, we see that when youth and adults interact with the opposite sub-population, any improvement in the removal parameters $\left(t_{i}\right)$ or decrease in activity parameters $\left(a_{i}\right)$ will reduce the reproduction number. 
A result for $\mathcal{R}_{0}$ that is independent of the mixing patterns is presented in Corollary 3.4.

Corollary 3.4: $\mathcal{R}_{0}=a_{i} /\left(\phi_{i}+\alpha_{i}\right)$, for any $i \in\{y, A\}$, if $\beta_{y A}=\beta_{A y}$, and $a_{2} /\left(\phi_{A}+\alpha_{A}\right)=$ $a_{1} /\left(\phi_{y}+\alpha_{y}\right)$.

Proof: Denote $\beta_{y A}=\beta_{A y}=\bar{\beta}$ and $a_{2} /\left(\phi_{A}+\alpha_{A}\right)=a_{1} /\left(\phi_{y}+\alpha_{y}\right)=K$. Note that $\beta_{y y}=$ $1-\beta_{y A}=1-\beta_{A y}=\beta_{A A}$, and let $\beta_{A A}=\beta_{y y}=\beta$. Then $R_{0}^{A A}=R_{0}^{y y}=K \beta$, and $R_{0}^{y A}=$ $R_{0}^{A y}=K \bar{\beta}$. Therefore, by substituting in (7) from Theorem 1: $\mathcal{R}_{0}=\frac{1}{2}\left[2 K \beta+\sqrt{4 K^{2} \bar{\beta}^{2}}\right]=$ $K(\beta+\bar{\beta})=K$.

\subsection{Homogeneous population stability analysis}

In this section, we present stability analysis of the interaction pattern within one of the sub-populations corresponding to like-with-like mixing. The initial conditions chosen for the simulation represent the arrival of an adult drug dealer into a susceptible community where there is no previous gang activity. In this case $G_{A}^{0}=1, G_{y}^{0}=0, S_{A}^{0}=N_{A}, S_{y}^{0}=N_{y}$, $R_{y}^{0}=R_{A}^{0}=0$. In the youth sub-population, like-with-like interaction will not produce a gang. We will then focus on stability analysis of the adult gang when $\mathcal{R}_{0}>1$.

The constant population assumption gives $S_{A}=N_{A}-G_{A}-R_{A}$. For simplicity of notation, we will assume that all variables and parameters refer to the adult sub-population and will not further use the $A$ subscript in the equations. Also, note that $\beta_{A A}=1, \beta_{y A}=\beta A y=$ 0 . The new simplified equations obtained from (5) and (6) are

$$
\begin{aligned}
\frac{\mathrm{d} G}{\mathrm{~d} t} & =a_{2}\left(\frac{N-G-R}{N}\right) G+\bar{f} \rho R-(\phi+\alpha) G, \\
\frac{\mathrm{d} R}{\mathrm{~d} t} & =\alpha G-\rho R .
\end{aligned}
$$

The nullcline equations obtained from (10) and (11) are

$$
\begin{aligned}
& R=\frac{-a_{2} G^{2}+\left(a_{2}-(\phi+\alpha)\right) N G}{a_{2} G-\bar{f} \rho N}, \\
& R=\frac{\alpha}{\rho} G .
\end{aligned}
$$

Solving (12)-(13) we obtain the gang-free trivial equilibrium, and the endemic equilibrium point defined as

$$
\begin{aligned}
& G^{*}=\rho \frac{N\left(a_{2}-\phi-\alpha(1-\bar{f})\right)}{a_{2}(\rho+\alpha)}, \\
& R^{*}=\alpha \frac{N\left(a_{2}-\phi-\alpha(1-\bar{f})\right)}{a_{2}(\rho+\alpha)} .
\end{aligned}
$$

We can linearize close to the equilibrium point and find the Jacobian of (10) and (11)

$$
J=\left(\begin{array}{cc}
\frac{-a_{2}}{N}(R+2 G)-(\phi+\alpha)+a_{2} & \frac{-a_{2}}{N} G+\bar{f} \rho \\
\alpha & -\rho
\end{array}\right) .
$$




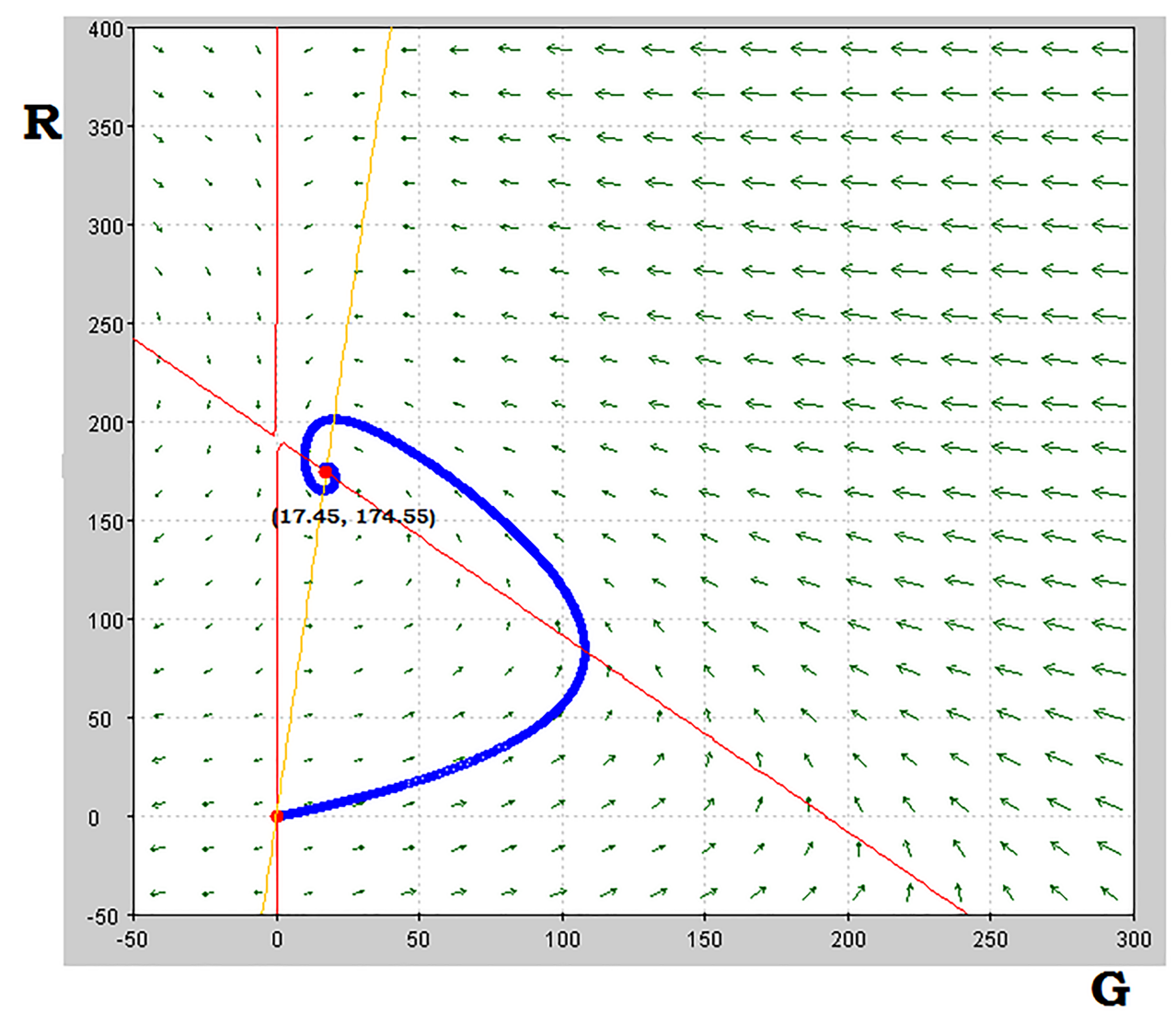

Figure 2. Orbit and endemic stable spiral equilibrium node in phase plane with intersecting nullclines.

The eigenvalues at equilibrium points $G^{*}, R^{*}$ were obtained for the constant parameters $P=a_{2}=5, N=300, \alpha=1, \phi=0.8, \rho=0.1, \bar{f}=0$ in our model. The trivial (gang-free) equilibrium is a saddle point with $\lambda_{1} \approx 3.22, \lambda_{2} \approx-0.52$. The endemic equilibrium $\left(G^{*} \approx\right.$ $\left.17.45, R^{*} \approx 174.55\right)$ is an asymptotically stable spiral node with $\lambda=-0.195 \pm 0.531 i$. The phase plane portrait in Figure 2 shows the solution field in the $G, R$ plane, nullclines, the orbit at our initial condition $G_{0}=1, R_{0}=0$, and the computed equilibrium.

The following is a result about the stability of the gang-free equilibrium for our model when parameters are given such that $\mathcal{R}_{0}>1$.

Theorem 3.5: The gang-free equilibrium for like-with-like mixing, is an unstable saddle point if $a_{2}-\phi>\alpha f$, and a stable node otherwise.

Proof: In (16) we substitute $G=R=0$. The simple matrix will have characteristic polynomial equation

$$
\lambda^{2}+\lambda\left(\rho+\phi+\alpha-a_{2}\right)+\rho\left(\phi+\alpha(1-\bar{f})-a_{2}\right)=0 .
$$

We denote $t=\phi+\alpha$. The two solutions of the quadratic equation are obtained from

$$
\lambda_{2}=\frac{1}{2}\left(-\left(\rho+t-a_{2}\right) \pm \sqrt{\left(\rho+\left(t-a_{2}\right)\right)^{2}-4\left(\rho\left(t-a_{2}\right)\right)+4 \alpha \bar{f} \rho}\right) .
$$


This simplifies to

$$
\lambda_{2}=\frac{1}{2}\left(-\left(\rho+t-a_{2}\right) \pm \sqrt{\left(\rho-\left(t-a_{2}\right)\right)^{2}+4 \alpha \bar{f} \rho}\right) .
$$

All parameters are positive, and solutions are real. Therefore, the solution to this quadratic equation renders a negative eigenvalue $\lambda_{1}$ by subtraction and a positive eigenvalue when $\left(\rho+\left(t-a_{2}\right)\right)<\left(\rho-\left(t-a_{2}\right)\right)^{2}+4 \alpha \bar{f} \rho$. This reduces to $\left(t-a_{2}\right)<\alpha \bar{f}$. Finally, take $t=\phi+\alpha$ and $1-\bar{f}=f$, to obtain the result. When the inequality is not met, then we obtain either two negative or one negative and a zero eigenvalue. In this instance the gang-free equilibrium is stable.

First of all, Theorem 3.5 says that as long as the activity of gang members minus the intervention parameter is larger than the product of the incarceration and rehabilitation parameter, the gang will spread to the community. Secondly, we observe in the proof, that the inequality $\left(t-a_{2}\right)<\alpha \bar{f}$ will be satisfied in our numerical simulations when $\mathcal{R}_{0}>1$ because the activity parameter is larger than the combined incarceration and social intervention ratios, i.e. $a_{2}>\phi+\alpha=t$. Therefore, the gang-free equilibrium is unstable when $\mathcal{R}_{0}>1$ under the initial conditions in our simulation.

Numerical results verify the stability and give us greater insight on the role of mixing patterns, parameters and the reproduction number.

\section{Numerical results}

The SGR gang model for youth and adult sub-populations was solved numerically for all three different mixing patterns. The floating parameters values have been obtained from a questionnaire handed to two ex-gang members and a former law enforcement agent. Therefore, the parameters are not obtained from published data, but only conform to the experience of our consultants. In Table 1, the parameters used for simulation purposes are summarized.

Initial conditions were chosen with no youth gang members, and one adult gang member entering a community with a large population of susceptible individuals such as housing projects in densely populated urban areas. The following graphs in Figures 3-5 show the results for different mixing patterns.

In all cases, we observe a non-zero endemic equilibrium. This can be interpreted as the continuous presence of gang activity in an established drug hot-spot. In Figure 3, the proportionate mixing pattern shows that approximately $78 \%$ of the youth population enters the gang compared to $53 \%$ of adults. In Figure 4, the preferred mixing gives a maximum of $79 \%$ of recruited youth and $49 \%$ of adults. The like-with-like mixing in Figure 5 is logically best under the initial condition that no youth has been involved in criminal activities in the past. It is then the best-case scenario that no youth will join the gang if they are protected from contact with adult gang members. In this case, a maximum of $38 \%$ of adults will be

Table 1. Parameters used in simulations.

\begin{tabular}{cccccccccccc}
\hline$N_{y}$ & $N_{A}$ & $a_{1}$ & $a_{2}$ & $\rho_{y}$ & $\rho_{A}$ & $\phi_{y}$ & $\phi_{A}$ & $\alpha_{y}$ & $\alpha_{A}$ & $f_{y}$ & $f_{A}$ \\
\hline 250 & 300 & 15 & 5 & 0.1 & 0.25 & 0.1 & 0.8 & 1 & 1 & 0.4 & 0.5 \\
\hline
\end{tabular}



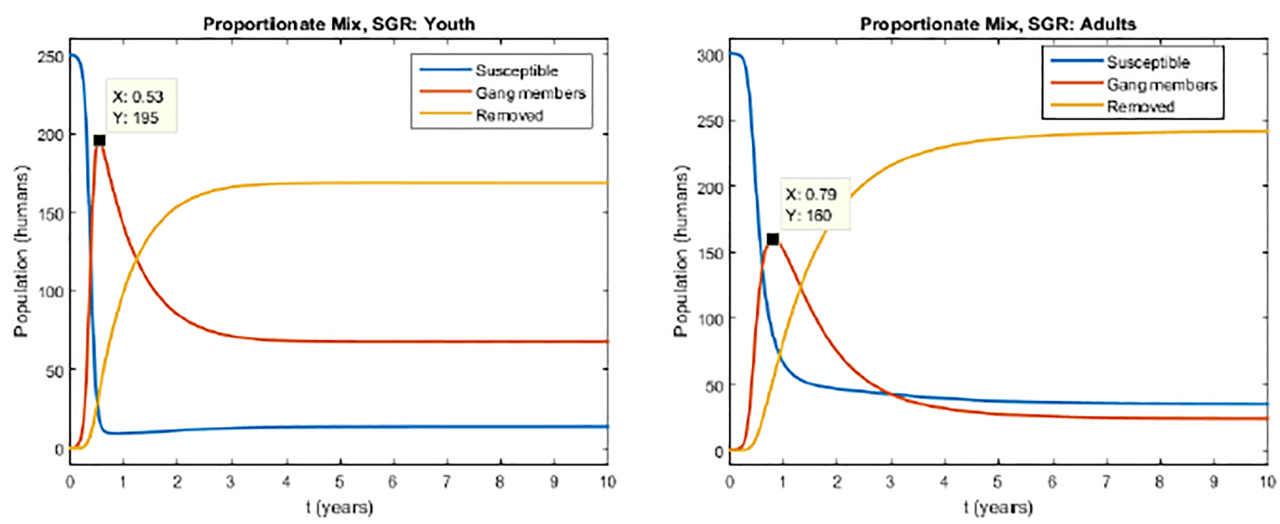

Figure 3. Proportionate mixing: random interaction among groups with higher gang recruitment in youth group.
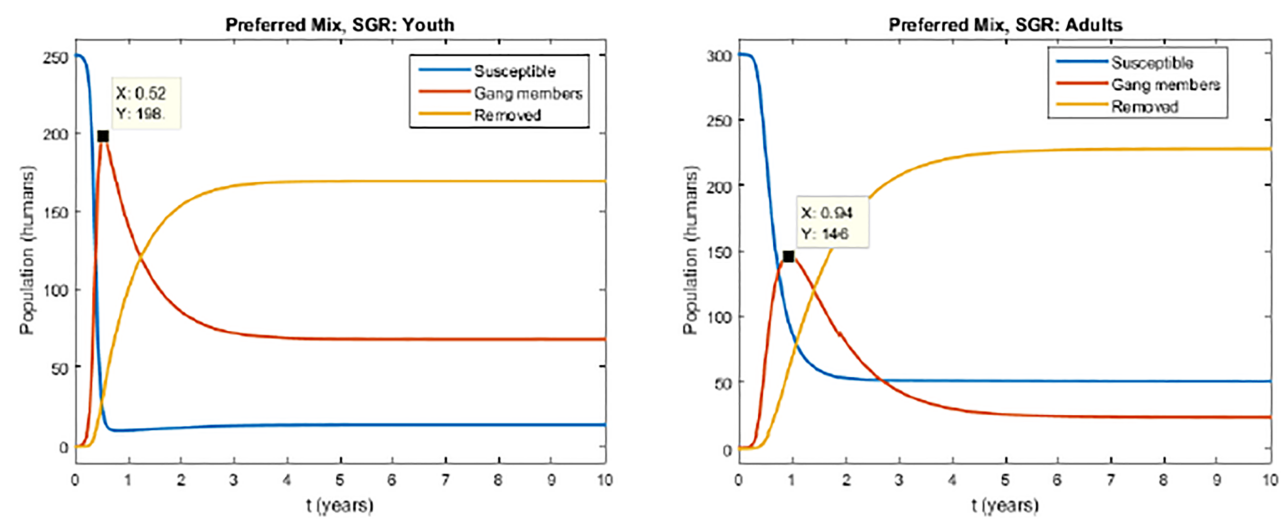

Figure 4. Preferred mixing with 50\% interaction within the homogeneous group shows a decrease in adult gang formation compared to proportionate mixing.
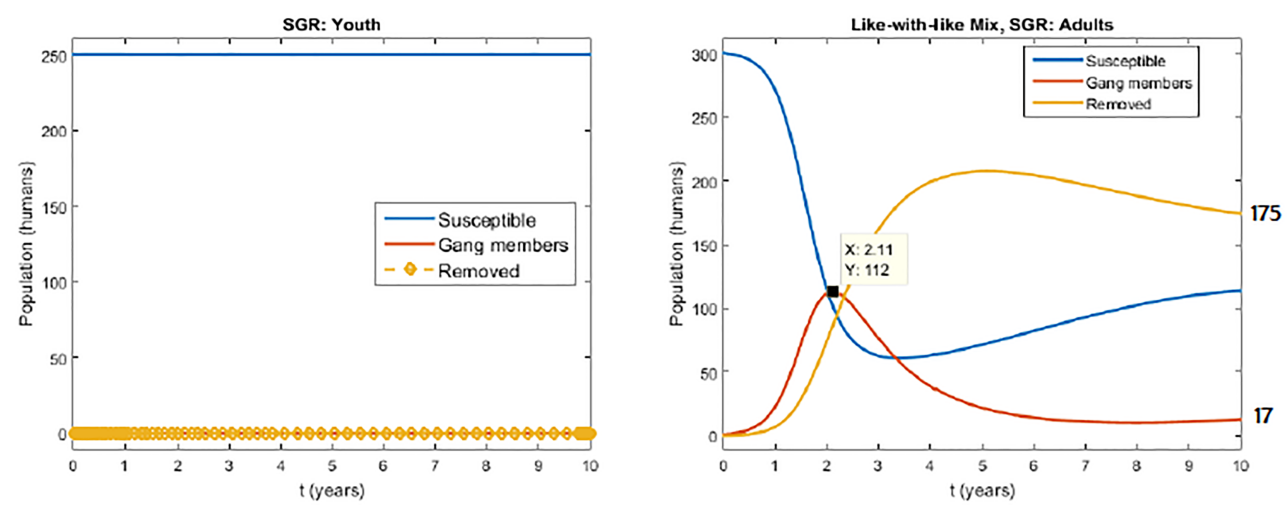

Figure 5. Like-with-like mixing shows zero-gang in youth and lowest adult gang recruitment. 
Table 2. Changes in $R_{0}$ with $\pi$.

\begin{tabular}{llllll}
\hline$\pi_{A}$ & 0.2 & 0.4 & 0.6 & 0.8 & 0.999 \\
$\mathcal{R}_{0}$ & 2.41 & 2.46 & 2.52 & 2.63 & 2.78 \\
\hline
\end{tabular}

recruited. The equilibrium point in Figure 5 matches the asymptotically stable spiral node obtained in the like-with-like stability analysis in Figure 2.

We have conducted numerous simulations to test what is the most favourable mixing pattern for gang formation. To visualize the sensitivity of the model to the differences in mixing interactions among the groups, we increase the $\pi$ parameter of preferred mixing in our simulations. A mixing pattern is considered ' favourable' for the development of a gang if the simulation results show a greater number of gang membership, and this peak is attained in a shorter time period. The numerical results have been instrumental in observing that, under our initial conditions and parameters, a larger $\mathcal{R}_{0}$ is not a good predictor of a bigger gang when modifying the sub-population interactions by changing the $\pi_{i}$ parameters. Numerical results show that the largest $a_{i}$ activity parameter determines the mixing pattern that favours gang formation because adult and youth gangs benefit from the interaction with the most active sub-population. This suggests that $a_{i}$ is a parameter with high sensitivity in our model. Details of the numerical simulations are discussed in the next paragraph.

In all simulations, the $\mathcal{R}_{0}$ increased as the $\pi_{A}$ value increased from proportional $\left(\pi_{A}=0\right)$ to like-with-like mixing $\left(\pi_{A}=1\right)$ as seen in Table 1 . Interestingly, it is not the case that gang formation favours like-with-like mixing, as suggested by an increase in $\mathcal{R}_{0}$. As before, we assume that an adult gangster enters a susceptible community with no previous criminal gang activities. The corresponding initial conditions for this simulation has one adult gang member $\left(G_{A}^{0}=1\right)$ and zero in the youth gang compartment $\left(G_{y}^{0}=0\right)$. We assume also that the youth population is much more active contacting others. In Figure 6, we observe a contrast in the behaviour of the adult and youth gangs when $\pi_{i}$ increases in preferred mixing. The $\mathcal{R}_{0}$ steadily increases with $\pi_{A}$, but the development of the adult gang slows down and decreases in size, opposite to the youth gang spread. Therefore, gang formation for adults favours proportional mixing, smaller $\pi_{A}$, rather than like-with-like. On the other hand, the youth gang favours interaction within its own group, higher $\pi_{y}$. The exception is the limit case when $\pi_{y}=1$, corresponding to like-with-like mixing, with zero youth gang members because of the initial condition $G_{y}^{0}=0$. We conclude that the largest activity parameter value $a_{i}$ under the initial conditions, determines the mixing pattern $\pi_{i}$ that favours gang spread of each population by increasing the interaction with the most active group. Therefore, an increasing $\mathcal{R}_{0}$ is not a sufficient indicator to determine the size of the gang. This result is in agreement with conclusions from Brauer (2008) that in models with heterogeneous mixing, the reproduction number is not sufficient to determine the size of the epidemic.

Drug trafficking data of gangs under investigation has not been available, and official data of past criminal activity that include minors is hard to obtain or incomplete for our purposes. Therefore, at this point, random noise was introduced to numerical results in order to create artificial experimental gang data. A parameter estimation algorithm has been tested for the youth contact parameter $a_{1}$, because the model exhibits large sensitivity to $a_{1}$ changes. A Nealder-Mead algorithm minimized the error successfully in Figure 7 

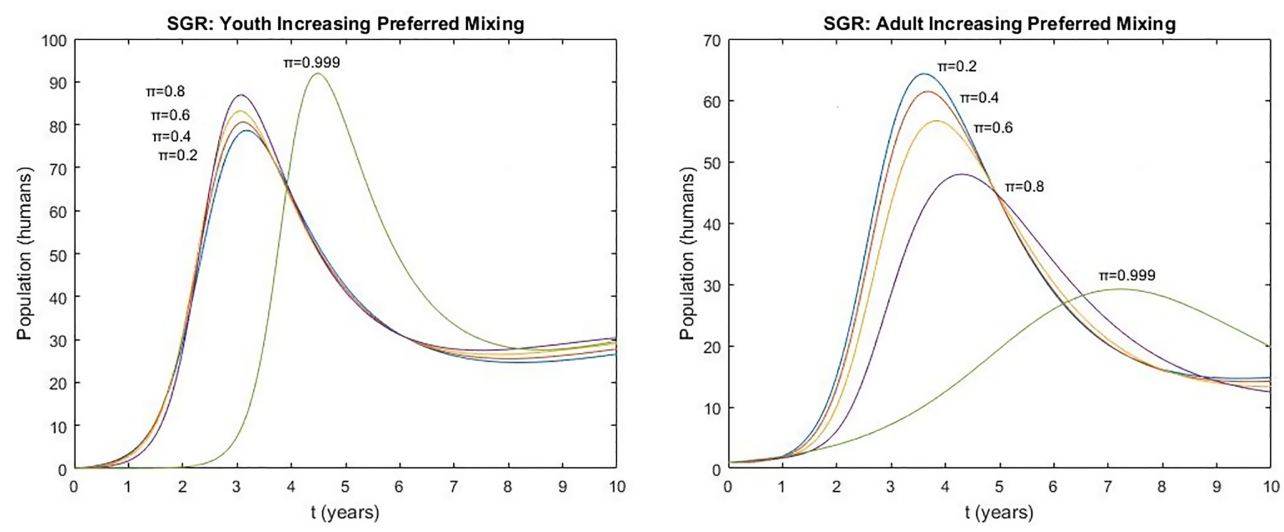

Figure 6. Opposite behaviour in youth vs. adult gangs as mixing becomes increasing homogeneous.

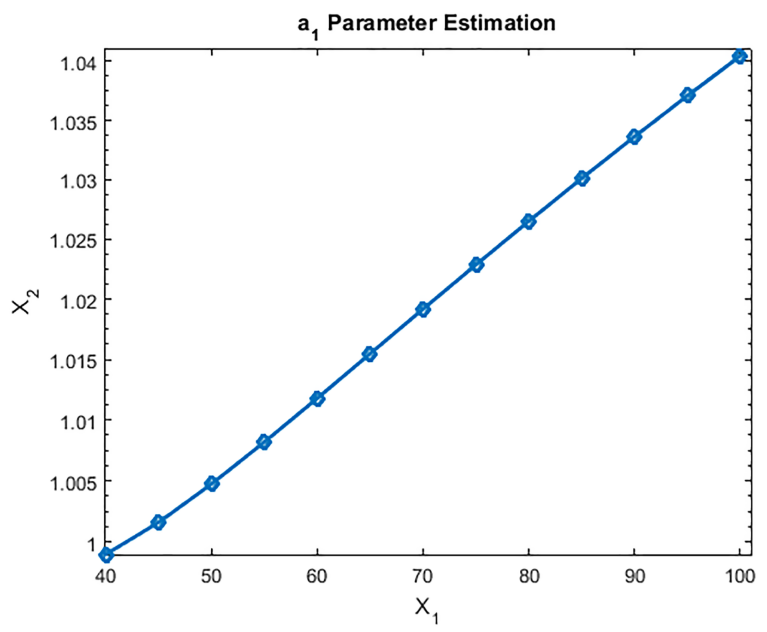

Figure 7. Parameter $a_{1}$ modified with $X_{1}=$ Noise in simulation, $X_{2}=a_{1}^{*} / a_{1}$, where $a_{1}^{*}$ is the parameter optimization result.

showing that the ratio between the estimated parameter and the actual parameter is close to one even when the introduced noise is of the order of $10^{2}$.

To adjust our simulation to the target size of an illegal-drug gang in a large susceptible housing project in the metropolitan area of San Juan, we modified the activity parameters $a_{i}$ and kept all other parameter constant. Results of this simulation are presented in Figure 8. This simulates the spread of a gang in a large susceptible population produced by introducing an adult gangster with no previous contacts in the neighbourhood. The results suggest that in order to develop a large gang of more than 100 youth and adults, a newcomer adult gangster will spend approximately three years. The simulation shows furthermore that the model has the capacity to produce insight for law enforcement by customizing the parameters such as $a_{i}$ to a targeted criminal activity data. These results will be confirmed in the Sensitivity analysis section. 

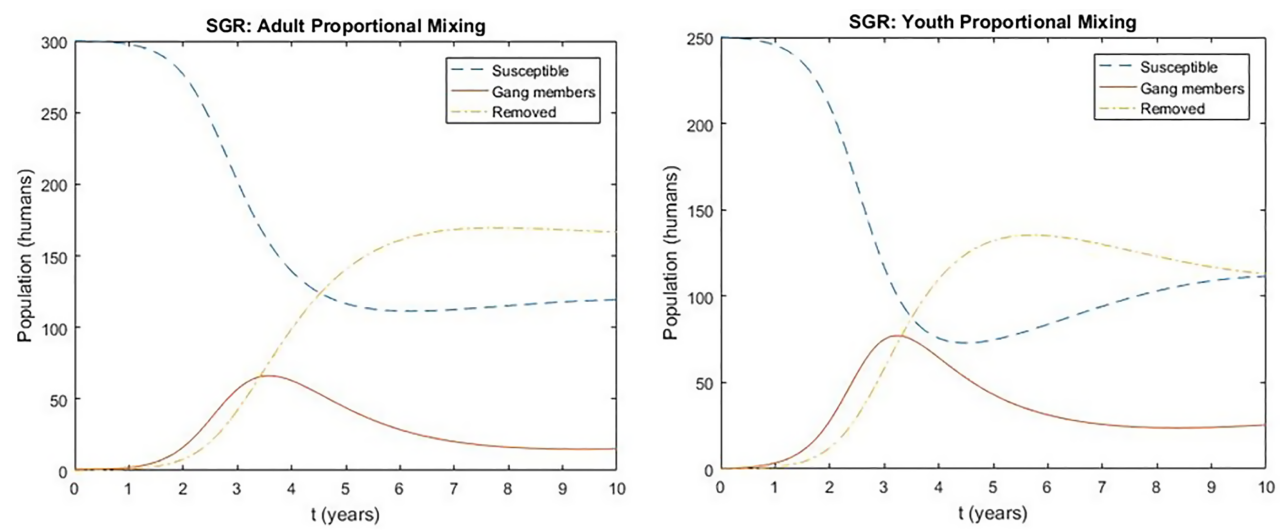

Figure 8. Optimizing $a_{i}$ parameter results to modify the size of a gang.

\section{Sensitivity analysis}

Numerical results showed sensitivity of the model to parameters $\pi, a_{1}$, in contrast to the information given by an increased $\mathcal{R}_{0}$ to measure gang spread. This motivated a forward sensitivity analysis of the model for preferred mixing that allows the modification of $\pi_{y}, \pi_{A}$ parameter observed in Figure 6. The sensitivity analysis of the preferred mixing model for 16 parameters is developed by computing the normalized forward sensitivity index, defined as

$$
\Upsilon_{p}^{u}:=\frac{\partial u}{\partial p} \times \frac{p}{u},
$$

for variable $u$ and parameter $p$ (Chitnis, Hyman, \& Cushing, 2008). The independent variables of the model are denoted as $G_{y}=x_{1}, R_{y}=x_{2}, G_{A}=x_{3}, R_{A}=x_{4}$, with $S_{y}=N_{y}-G_{y}-R_{y}$ and $S_{A}=N_{A}-G_{A}-R_{A}$. Therefore, the equations for this section simplify to

$$
\begin{aligned}
\frac{\mathrm{d} x_{1}}{\mathrm{~d} t} & =a_{1}\left(N_{y}-x_{1}-x_{2}\right)\left[\left(\frac{\beta_{y y} x_{1}}{N_{y}}\right)+\left(\frac{\beta_{y A} x_{3}}{N_{A}}\right)\right]+\bar{f}_{y} \rho_{y} x_{2}-x_{1}\left(\phi_{y}+\alpha_{y}\right), \\
\frac{\mathrm{d} x_{2}}{\mathrm{~d} t} & =\alpha_{y} x_{1}-\rho_{y} x_{2}, \\
\frac{\mathrm{d} x_{3}}{\mathrm{~d} t} & =a_{2}\left(N_{A}-x_{3}-x_{4}\right)\left[\left(\frac{\beta_{A A} x_{3}}{N_{A}}\right)+\left(\frac{\beta_{A y} x_{1}}{N_{y}}\right)\right]+\bar{f}_{A} \rho_{A} x_{4}-x_{3}\left(\phi_{A}+\alpha_{A}\right), \\
\frac{\mathrm{d} x_{4}}{\mathrm{~d} t} & =\alpha_{A} x_{3}-\rho_{A} x_{4} .
\end{aligned}
$$

We disregard dependent parameters: $\beta_{y A}=1-\beta_{y y}, \beta_{A y}=1-\beta_{A A}$, and note that $\beta_{y y}, \beta_{A A}$ are functions of $\pi_{y}, \pi_{A}, N_{y}, N_{A}, a_{1}, a_{2}$, for calculation of total derivatives. The 16 parameters included in this study are $\mathrm{P}=\left[\beta_{y y}, \pi_{y}, a_{1}, f_{y}, \rho_{y}, \phi_{y}, \alpha_{y}, N_{y}, \beta_{A A}, \pi_{A}, a_{2}, f_{A}\right.$, $\left.\rho_{A}, \phi_{A}, \alpha_{A}, N_{A}\right]$. 
Table 3. Model parameter values for the endemic equilibrium solution used as baseline.

\begin{tabular}{llcccccc}
\hline$\beta_{y y}$ & $\pi_{y}$ & $a_{1}$ & $f_{y}$ & $\rho_{y}$ & $\phi_{y}$ & $\alpha_{y}$ & $N_{y}$ \\
0.88 & 0.5 & 20 & 0.1 & 0.4 & 0.6 & 1 & 250 \\
\hline$\beta_{A A}$ & $\pi_{A}$ & $a_{2}$ & $f_{A}$ & $\rho_{A}$ & $\phi_{A}$ & $\alpha_{A}$ & $N_{A}$ \\
0.62 & 0.5 & 5 & 1 & 0.1 & 0.8 & 1 & 300 \\
\hline
\end{tabular}

Table 4. Sensitivity indices for variables to all parameters at the endemic equilibrium, using parameter values in Table 3 corresponding to the numerical endemic equilibrium solution.

\begin{tabular}{lrrrr}
\hline & $G_{y}$ & $R_{y}$ & \multicolumn{1}{c}{$G_{A}$} & \multicolumn{1}{c}{$R_{A}$} \\
\hline$\beta_{y y}$ & 0.029 & 0.030 & 0.004 & 0.004 \\
$\pi_{y}$ & 0.001 & 0.001 & -0.023 & -0.023 \\
$a_{1}$ & 0.044 & 0.045 & 0.029 & 0.029 \\
$f_{y}$ & -0.006 & -0.006 & -0.001 & -0.001 \\
$\rho_{y}$ & 0.709 & -0.285 & 0.096 & 0.095 \\
$\phi_{y}$ & -0.034 & -0.034 & -0.005 & -0.005 \\
$\alpha_{y}$ & -0.720 & 0.282 & -0.098 & -0.096 \\
$N_{y}$ & 0.999 & 1.007 & 0.023 & 0.022 \\
$\beta_{A A}$ & 0.000 & 0.000 & -0.160 & -0.157 \\
$\pi_{A}$ & 0.003 & 0.003 & -0.077 & -0.075 \\
$a_{2}$ & -0.003 & -0.003 & 0.171 & 0.168 \\
$f_{A}$ & 0.000 & 0.000 & -0.106 & -0.105 \\
$\rho_{A}$ & 0.001 & 0.001 & 0.805 & -0.209 \\
$\phi_{A}$ & 0.000 & 0.000 & -0.084 & -0.082 \\
$\alpha_{A}$ & -0.001 & -0.001 & -0.895 & 0.103 \\
$N_{A}$ & -0.003 & -0.003 & 0.997 & 0.979 \\
\hline
\end{tabular}

Denoting the right-hand side of Equations (17)-(20) as $g_{1}-g_{4}$, we find the full derivative at equilibrium of equation $k$ with respect to parameter $j$ :

$$
\frac{\mathrm{d} g_{k}}{\mathrm{~d} p_{j}}=\sum_{i=1}^{4}\left(\frac{\partial g_{k}}{\partial x_{i}} \frac{\partial x_{i}}{\partial p_{j}}\right)+\sum_{l=1}^{16}\left(\frac{\partial g_{k}}{\partial p_{l}} \frac{\partial p_{l}}{\partial p_{j}}\right)=0
$$

for $1 \leq k \leq 4$ and $1 \leq j \leq 16$. Therefore, we obtain for the $j$ th parameter a system of four equations of the form:

$$
\sum_{i=1}^{4} \frac{\partial g_{k}}{\partial x_{i}} \frac{\partial x_{i}}{\partial p_{j}}=-\sum_{l=1}^{16} \frac{\partial g_{k}}{\partial p_{l}} \frac{\partial p_{l}}{\partial p_{j}}
$$

and solve this system defined by the 4 by 4 Jacobian matrix $A=\left[a_{k, i}\right], 1 \leq k, i \leq 4$, with $a_{k, i}=\partial g_{k} / \partial x_{i}$, for the sensitivity vector of each variable to the parameter $p_{j}$, given by $\left[\partial x_{1} / \partial p_{j}, \ldots, \partial x_{4} / \partial p_{j}\right]^{\mathrm{T}}$.

We computed the normalized sensitivity index of the preferred mixing model to all 16 parameters, for the numerical endemic equilibrium $x^{*}=[69,171,22,224]$ obtained from our numerical solution with its corresponding parameter values $P^{*}$ in Table 3 that are used as baseline. The results of the sensitivity index for each variable $G_{y}, R_{y}, G_{A}, R_{A}$ presented in Table 4.

The sensitivity analysis results are very enlightening. We first observe that the direction given by the sign of each sensitivity index seems to correctly describe an increase or 
decrease in the gang $\left(G_{i}\right)$ and removed $\left(R_{i}\right)$ variables. For the $G_{i}$, we see that the rehabilitation $\left(f_{i}\right)$, intervention $\left(\phi_{i}\right)$, and removal $\left(\alpha_{i}\right)$ rates are negative, as expected, decreasing the number of gang members in both populations. An increase in the number of susceptible individuals $\left(N_{i}\right)$ always produces an increase in its sub-population. Nonetheless, an interesting change of sign occurs when $N_{A}$ increases. It might be expected that all variables increase with the susceptible population, but this is not the case for the youth variables as $N_{A}$ increase. A higher $N_{A}$ produce an increase in the probability of random interactions of youth with the less active adult group, decreasing $G_{y}, R_{y}$. We ran again the sensitivity analysis code changing the preferred mixing from $\pi_{y}=0.5$ to $\pi_{y}=0.9$ to test this hypothesis. The sensitivity indices of youth variables to the $N_{A}$ parameter reduced to zero. This shows that an increase of $N_{A}$ decreases the youth population when a larger proportion of interactions are random $\left(\pi_{y}=0.5\right)$, but have negligible effects if the youth prefer to interact more within its own group $\left(\pi_{y}=0.9\right)$. This is a consequence of the effect that the larger activity parameter $a_{2}=20$ has on the spread of the gang. Therefore, a higher probability of interacting with youth will increase the spread of the gang, while otherwise, a higher probability of interaction with adults decrease the active youth group variables.

Regarding the magnitude of the parameters, the highest positive index is the population parameter $\left(N_{i}\right)$ for its corresponding sub-population. The removal rate $\left(\alpha_{i}\right)$ is the parameter that decreases most of the corresponding variables. The release parameter $\left(\rho_{y}\right)$ is the one that reduces the most the number of removed individuals, and, in the opposite direction, incarceration/removal rate $\left(\alpha_{i}\right)$ contributes the most to the number of removed individuals in both populations. The intervention rate $\left(\phi_{y}\right)$ has a higher impact in reducing the $G_{y}$ than the rehabilitation rate $\left(f_{y}\right)$, but in the adult sub-population it is exactly the opposite. This responds to the larger $f_{A}=1>f_{y}=0.1$. This was also verified by changing this parameter to $f_{A}=0.1$ and the new sensitivity indices of both $G_{A}$ and $R_{A}$ to $f_{A}(-0.021)$ are smaller in magnitude to those for $\phi_{A}(-0.167)$. The influence of the higher activity youth subpopulation is also observed in the intervention $\left(\phi_{y}\right)$ and rehabilitation $\left(f_{y}\right)$ parameters on the adult variables. The youth group decrease the adult variables, $G_{A}$ and consequently $R_{A}$, to an expected lesser extent. The influence of the corresponding adult parameters on the youth variables is negligible. This is understood again in light of the lower activity parameter of adults $\left(a_{2}=5\right)$, and therefore a smaller influence on the spread of the gang.

The sensitivity of the model to the activity parameters $a_{i}$, and interaction $\pi_{i}, \beta_{i} i$ can be understood in the same light as the previous discussion. A higher youth interaction in preferred mixing driven by the $\left(p i_{y}\right)$ parameter benefits the spread of the youth gang, but not the adult. This is also confirmed with the sensitivity to $\pi_{A}$. The sensitivity indices for the $\beta_{i} i$ parameters are also a consequence of the most large activity of the youth subpopulation. Here, the $\beta_{y y}$ parameter changes will increase all variables, as this produces a larger interaction among the most active youth sub-population. The opposite is true for the $\beta_{A A}$. An increase of the proportion of adult-with-adult interaction will decrease the spread of the adult gang. Again the increase of the activity of the youth group $\left(a_{1}\right)$ increases all variables. Nonetheless, an increase in the adult activity $\left(a_{2}\right)$ improves the adult variables but will slightly decrease the youth variables. An argument similar to the $N_{A}$ parameter sensitivities explains how an increased probability of interaction with less active $G_{A}$ will decrease the youth $\beta_{y y}$ parameter and variables. 


\section{Conclusion}

We have developed a model with six variables for the spread of a youth gang in a heterogeneous population by age groups. Three mixing patterns have been used to govern interaction between the groups. Some parameters have been introduced that enable the study of the effects of social interventions to persuade criminals to leave the gang, recidivism vs. rehabilitation, detention/incarceration, activity and population for youth and adults. These parameters have been approximated from census data, ex-gang members questionnaire, and the collaboration of a former law-enforcement agent. We have presented formulations for the reproduction number, stability analysis for homogeneous mixing, numerical results, and sensitivity analysis for the model. A parameter estimation algorithm shows that this model can be tailored to law enforcement data, producing insight into the dynamics of gang spread among youth, a complex social problem that is deforming the vulnerable kids in PR and other nations.

The mathematical analysis of the model presents the formulation for the reproduction number and its simplified forms for different mixing patterns that include like-with-like and proportionate mixing. An analysis of the reproduction number $\mathcal{R}_{0}$ and sensitivity analysis showed that larger educational and counselling intervention parameter will also decrease the intensity of gang recruitment. For the homogeneous like-with-like mixing, we have presented formulas to obtain the endemic equilibrium, and showed the conditions for the gang-free equilibrium to be an unstable saddle point in the like-with-like mixing when $\mathcal{R}_{0}>1$. The phase plane for the initial conditions and parameters of the simulation have shown that the endemic equilibrium is an asymptotically stable spiral node. This result has been confirmed in the numerical simulation results.

The numerical simulations study the formation of a large gang in a susceptible population of youth and adults initiated by a recruiting adult with no previous connections in the neighbourhood. Numerical results for $\mathcal{R}_{0}>1$ shown in Figure 5 confirmed a nonzero endemic equilibrium that is consistent with the phase plane results obtained for the like-with-like mixing. proportionate and preferred mixing in Figures 3 and 4 also show and endemic equilibrium that is consistent with the existence of drug hot-spots. These places where drug-related gang activities have been initiated continue to be drug dealing location in time because different gangs will takeover the illegal business at the spot. After a previous gang in the hot-spot has been dismantled, the successor gang sometimes chooses to keep the same name. A parameter estimation algorithm for the activity parameter $a_{i}$ has been developed to best fit the model to a scenario of a law-enforcement investigation. The sensitivity of the model to the activity parameter has given insight on the time needed to develop a large gang under the initial condition of a single recruiting adult. This parameter is also the prime predictor of the most favourable mixing pattern for youth and adults gang formation showing that an increasing $\mathcal{R}_{0}$ is not sufficient for this purpose.

The sensitivity analysis showed that the sub-population with the largest activity parameter will drive the spread of the gang. Therefore, interventions, rehabilitation, and removal efforts should be focused on this group in order to decrease the spread of a gang in a susceptible community. It also shows the size of the susceptible communities will promote the spread of a gang. Considering the above, some helpful strategies might include the building of smaller housing projects. Also, a removal strategy that promotes moving susceptible 
families to different communities might prove to be helpful to deter the local spread of a gang that is considered in this model.

An idealistic conclusion can be supported by the numerical results of the simulations that encourage the involvement of concerned citizens and youth organizations. If young kids with no previous gang involvement do not interact with gang recruiting adults, then simultaneously the adult gang will not prosper quickly and kids will not be involved in drug trafficking. The like-with-like mixing pattern shows that the best course of action to curtail the spread of a gang is prevention. Therefore, investments in programmes that develop an interest in arts, music and sports, recreation and other after-school activities continue to be the best resources to rescue vulnerable young people from the long-term consequences of crime.

\section{Disclosure statement}

No potential conflict of interest was reported by the authors.

\section{References}

Brauer, F. (2008). Epidemic models with heterogeneous mixing and treatment. Bulletin of Mathematical Biology, 70(7), 1869-1885.

Chakraborty, B. (2018, March 1). Puerto Rico turns to DOJ amid escalating post-Maria drugfueled violence. Retrieved from https://www.foxnews.com/us/-puerto-rico-turns-to-doj-amidescalating-post-maria-drug-fueled-violence

Chitnis, N., Hyman, J. M., \& Cushing, J. M. (2008). Determining important parameters in the spread of malaria through sensitivity analysis of a mathematical model. Bulletin of Mathematical Biology, 70, 1272-1296. doi:10.1007/s11538-008-9299-0

Choe, S., \& Lee, S. (2015). Modeling optimal treatment strategies in a heterogeneous mixing model. Theoretical Biology and Medical Modelling, 12(1), 28.

van den Driessche, P., \& Watmough, J. (2002). Reproduction numbers and sub-threshold endemic equilibria for compartmental models of disease transmission. Mathematical Biosciences, 180, $29-48$.

Kermack, W. O., \& McKendrick, A. G. (1991a). Contributions to the mathematical theory of epidemics - I. The problem of endemicity. Bulletin of Mathematical Biology, 53(1-2), 33-55.

Kermack, W. O., \& McKendrick, A. G. (1991b). Contributions to the mathematical theory of epidemics - II. The problem of endemicity. Bulletin of Mathematical Biology, 53(1-2), 57-87.

Levantesi, S. (2018, April 30). A reduced police force faces rising crime. Retrieved from http://periodismoinvestigativo.com/2018/04/una-fuerza-policiaca-reducida-enfrenta-elaumento-en-la-criminalidad-en-puerto-rico/

McMillon, D., Simon, C. P., \& Morenoff, J. (2014). Modeling the underlying dynamics of the spread of crime. PLoS ONE, 9(4), e88923.

Mohammad, F., \& Roslan, U. A. M. (2017, August). Analysis on the crime model using dynamical approach. AIP conference proceedings (Vol. 1870, No. 1, p. 040067). AIP Publishing.

Muñoz-González, R. (2007). Study of risk factors and its intensity associated to minors assigned to the first court of first instance: 2007 fiscal year. Court Administration Office, San Juan, PR.

Office of Court Administration. Minor's Court. Government of PR. (2018). Retrieved from http://www.ramajudicial.pr/orientacion/menores.htm

Office of Youth Affairs. (2002). Analysis of delinquency among youth in Puerto Rico. Governor's Office, Commonwealth of Puerto Rico, San Juan, PR.

Padmanabhan, P., Seshaiyer, P., \& Castillo-Chavez, C. (2017). Mathematical modeling, analysis and simulation of the spread of Zika with influence of sexual transmission and preventive measures. Letters in Biomathematics, 4(1), 148-166. 
Rey-Hernandez, C., \& Hernandez-Angueira, L. (2014). Human trafficking: Modern slavery in Puerto Rico. Ricky Martin Foundation. UPR, Rio Piedras Campus, San Juan, PR.

Silverstein, J. (2018, August 28). Hurricane Maria is now one of the deadliest disasters in U.S. history. Retrieved from https://www.cbsnews.com/news/puerto-rico-hurricane-maria-is-now-one-ofthe-deadliest-disasters-in-u-s-history

Simon, C. P., \& Jacquez, J. A. (1992). Reproduction numbers and the stability of equilibria of SI models for heterogeneous populations. SIAM Journal on Applied Mathematics, 52(2), 541-576.

Sooknanan, J., Bhatt, B., \& Comissiong, D. M. G. (2013). Catching a gang - a mathematical model of the spread of gangs in a population treated as an infectious disease. International Journal of Pure and Applied Mathematics, 83(1), 25-43.

Torres-Gotay, B. (2017, March 26). The phenomenon of criminal children. Retrieved from https://www.elnuevodia.com/noticias/seguridad/nota/elfenomenodelosninoscriminales2304544/ 